Sādhanā Vol. 39, Part 4, August 2014, pp. 843-858. (C) Indian Academy of Sciences

\title{
Give-and-take based peer-to-peer content distribution networks
}

\author{
SAURABH AGGARWAL ${ }^{1, *}$, JOY KURI ${ }^{1}$ and CHANDAN SAHA ${ }^{2}$ \\ ${ }^{1}$ Department of Electronic Systems Engineering, Indian Institute of Science, \\ Bangalore 560 012, India \\ ${ }^{2}$ Department of Computer Science and Automation, Indian Institute of Science, \\ Bangalore 560 012, India \\ e-mail: saggarwal@cedt.iisc.ernet.in; kuri@cedt.iisc.ernet.in; \\ chandan@csa.iisc.ernet.in
}

MS received 23 April 2013; revised 12 February 2014; accepted 25 March 2014

\begin{abstract}
Content Distribution Networks (CDNs) are widely used to distribute data to large number of users. Traditionally, content is being replicated among a number of surrogate servers, leading to high operational costs. In this context, Peer-to-Peer (P2P) CDNs have emerged as a viable alternative. An issue of concern in P2P networks is that of free riders, i.e., selfish peers who download files and leave without uploading anything in return. Free riding must be discouraged. In this paper, we propose a criterion, the Give-and-Take $(G \& T)$ criterion, that disallows free riders. Incorporating the G\&T criterion in our model, we study a problem that arises naturally when a new peer enters the system: viz., the problem of downloading a 'universe' of segments, scattered among other peers, at low cost. We analyse this $\mathcal{N} \mathcal{P}$-hard problem, and characterize the optimal download cost under the G\&T criterion. We propose an optimal algorithm, and provide a sub-optimal algorithm that is nearly optimal, but runs much more quickly; this provides an attractive balance between running time and performance. Finally, we compare the performance of our algorithms with that of a few existing P2P downloading strategies in use. We also study the computation time for prescribing the strategy for initial segment and peer selection for the newly arrived peer for various existing and proposed algorithms, and quantify cost-computation time trade-offs.
\end{abstract}

Keywords. Peer-to-peer networks; algorithms.

\section{Introduction}

A Content Distribution Network (CDN) contains copies of data placed at various surrogate servers in the network so that users across the world can access the content (figure 1). The problem

*For correspondence 
of scaling CDNs with the number of users is an important issue (Vakali \& Pallis 2003). Proposed solutions were observed to be economically infeasible (Choffnes \& Bustamante 2008; Plissonneau et al 2005).

The peer-to-peer network emerged as an alternative for single source data networks that can work over low bandwidth links. Compared to traditional content distribution schemes, peerto-peer networks permit significant reduction in the distributor's hardware, bandwidth resource costs and reduce the dependence on the original distributor (Menasché et al 2010), as these costs are shared among users who desire to download files.

In a peer-to-peer content distribution network (P2PCDN), each user downloads segments of files from other users (mostly) and is expected to upload to other users as well (figure 2). The peers are also encouraged to exchange among themselves as the bandwidth cost at the surrogate server is usually high (Plissonneau et al 2005).

However, most practical P2PCDN systems face difficulties because of free riders. These are selfish peers who download files, uploading negligible data or nothing in return (Feldman \& Chuang 2005; Feldman et al 2006; Typpi 2009; Karakaya et al 2009). P2P networking is based on the idea of willing participation of peers, and free riders violate this principle. For effective operation of P2PCDNs, free riding must be discouraged.

Various policies and methods have been proposed (Feldman et al 2006; Feldman \& Chuang 2005; Locher et al 2006; Rahman et al 2010; Nishida \& Nguyen 2010; Karakaya et al 2009) to discourage free riding. All these policies have weaknesses that can be exploited. For example, in the whitewashing attack (Feldman et al 2006), a peer assumes different identities, and pretending to be a new peer downloads some segments without uploading anything in return. Over time, a malicious user can accumulate substantial amount of data by exploiting the system. Peers can also falsify their reputation values and spoof identities for their benefit (Karakaya et al 2009).

In this paper, we propose the Give-and-Take criterion $(G \& T)$, which prohibits free riding. Any existing policy can be augmented to incorporate the G\&T criterion. Essentially, the idea is that a peer $A$ can download segments from another peer $B$ if and only if $A$ offers at least

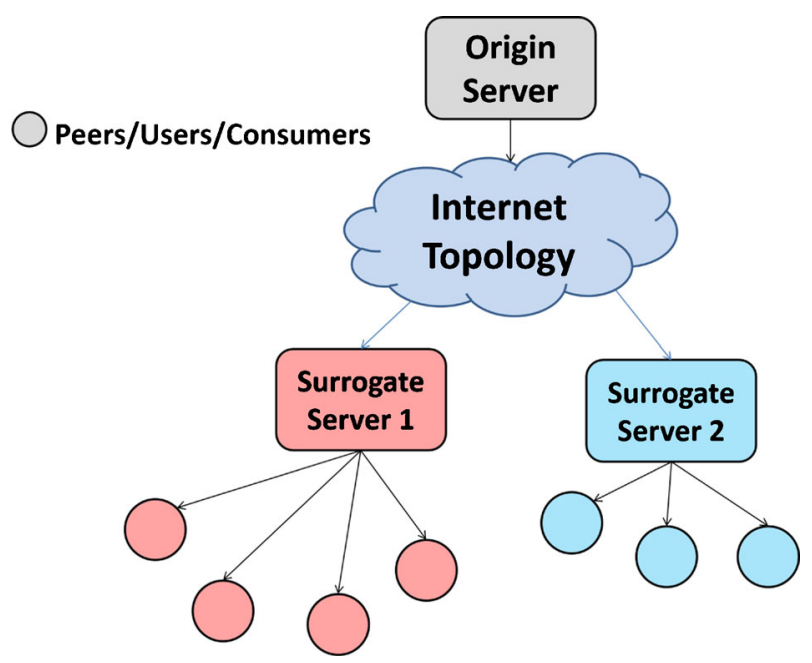

Figure 1. Content distribution network. 


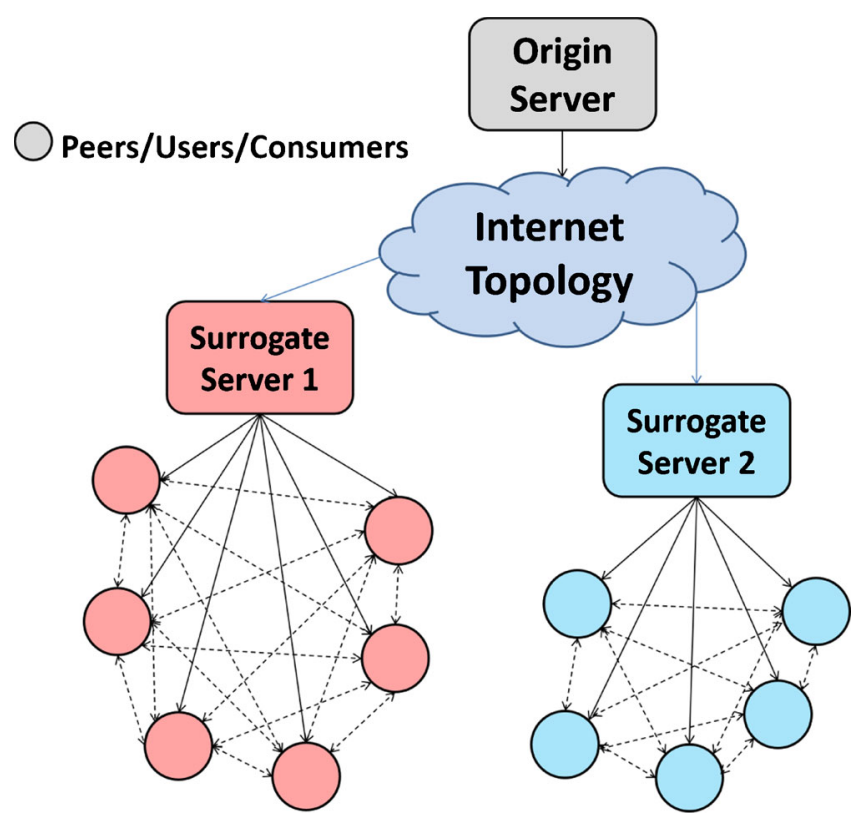

Figure 2. Peer-to-peer content distribution network (enabling exchange between peers decreases the load on surrogate server which allows more users to connect to surrogate server).

one segment for $B$ to download. In this work, we consider the special case in which a peer is willing to trade all the segments it has in exchange for even one segment that he does not have. In other words, peers 'give liberally,' even if they take just one new segment. Because of this, after exchange, each peer possesses a segment set that is the union of the segment sets before exchange.

The G\&T criterion does not depend on identity of the peers involved. To meet the criterion, peer A must offer at least one segment to peer B, and vice versa. The criterion remains unaffected even if $\mathrm{A}$ is the mutated identity of some other peer. This is why a whitewasher derives no benefit: even with a changed identity, a whitewasher had provided some segments in return to the other peer. Hence, a whitewasher cannot keep downloading small amounts and accumulate segments.

Incorporating the G\&T criterion in our model, we study a problem that arises naturally when a new peer (namely, peer 0) enters the system - the problem of downloading a 'universe' of segments, scattered among other peers, at least cost. The cost of exchange between peers 0 and $j$ is fixed. The cost can depend on a number of factors such as the delay incurred in completion of exchanges (with higher delays implying higher costs). Reputation of the peers can also influence cost; for example, if peer $j$ has a history of selfishness, a high cost can be assigned — this discourages exchanges with $j$ and serves as a punitive measure to peer $j$ for selfishness.

Peer 0 has no segments with him, the G\&T criterion implies that exchanges with peers cannot even begin. To get started, peer 0 downloads some segments from the seed or surrogate server, which is a storehouse of all segments. We note that the seed is not a peer, and the $\mathrm{G}$ and $\mathrm{T}$ criterion does not apply to downloading of segments from the seed. Peer 0's overall objective is to acquire the universe of segments at low aggregate cost. However, the cost of downloading from the seed is very high. Thus, peer 0's strategy would be to download a few segments from the seed such that G\&T compliant exchanges with peers can begin. For the purpose of analysis 
in this paper, we assume that system is changing at a slow rate, effectively making it static. If the system is more dynamic, given the current state of the system, the analysis in this paper can be used to find the best possible action (for example, the decision to join the network or not) at that instant.

We propose an optimal algorithm to solve this problem. We compare the performance of a few commonly used P2P downloading strategies with the optimal algorithm.

This paper makes the following contributions. Firstly, we propose a criterion called Give and Take (G\&T) that prohibits free riding. Secondly, we formulate and solve a problem where a new peer wishes to download a universe of objects at low cost, while satisfying the G\&T criterion at each exchange.

Section 2 discusses the system model in detail, followed by an analysis of the problem, optimal and sub-optimal algorithms in section 3. Section 4 compares the results with other algorithms used in P2P networks.

\section{System model}

We consider a scenario with $m$ peers, a newly arrived peer, denoted 'peer 0' and one Seed (or Surrogate server). The objective of peer 0 is to collect the universe at minimum cost. The cost of fetching the segment set with peer $j$ is $C_{j}$.

\subsection{Notation}

- Complete segment set: $N=\{1,2, \cdots n\}$

- Peer set: $M=\{1,2, \cdots m\}$

- Segment container sets: Segments with peer $j$

$$
O_{j}=\{i \in N \text { : peer } j \text { has segment } i\} \forall j \in M
$$

- $\left[P, C_{\text {opt }}\right]=\operatorname{setcover}\left(N_{s u b}, M_{s u b}, C\left(M_{s u b}\right)\right)$

setcover is a function with the following parameters:

$N_{\text {sub }}$ : Set of segments which needs to be fetched; $N_{s u b} \subseteq N$

$M_{s u b}$ : Subset of peers; $M_{s u b} \subseteq M$

$C\left(M_{\text {sub }}\right)$ : Cost vector for peers in $M_{\text {sub }}$

$P$ : Set of peers providing a minimum cost set cover, $P \subseteq M_{s u b}$ and $\cup_{j \in P} O_{j}=N_{s u b}$

$C_{o p t}$ : Cost of a minimum cost set cover

The objective of the set cover is to select a collection of minimum cost subsets such that each element of the universe is present in at least one of the subsets. There may be several collections $(P)$ which cover the universe at the least cost. Computation of minimum cost set cover is a $\mathcal{N} \mathcal{P}$-hard problem (Caprara et al 2000), but various approximation algorithms are available (Caprara et al 2000; Chavatal 1979; Feige 1998; Alon et al 2006).

\subsection{Assumptions}

A1: The cost of fetching any segment from the seed is same, and is much higher than the cost of fetching the segment from a peer. This assumption is made as usually segments are of the same size, and the bandwidth cost at the surrogate server is high (Aggarwal et al 2007). 


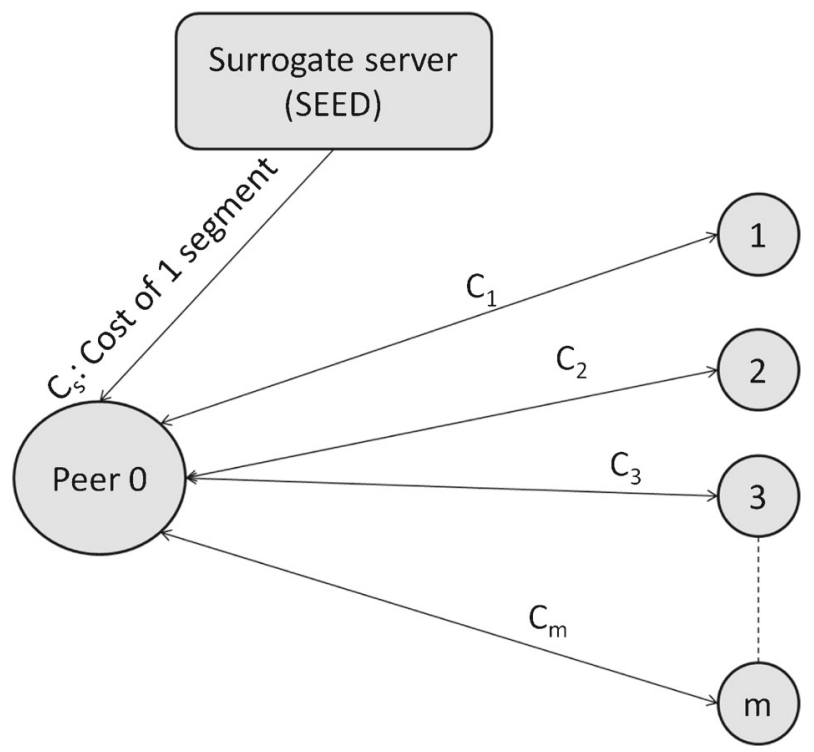

Figure 3. Cost structure for peer 0 .

The surrogate server has to serve a large number of users, which leads to increased cost of resources at the surrogate server tremendously (figure 3):

$$
C_{s}(e)=C_{s}>>C_{j} \quad \forall e \in N \text { and } j \in M
$$

A2: Peer 0 does not have any segment when it enters the system.

A3: Give-and-Take $(\mathbf{G \& T})$ criterion $\quad$ Peer 0 will be willing to exchange with peer $j$ if peer $j$ has at least one segment which is unavailable with peer 0. Similarly, peer $j$ will be willing to exchange with peer 0 iff peer 0 has a segment which is unavailable with peer $j$. Let peer 0 have $O_{0}$ as the set of segments at the time of exchange with peer $j$. Then following must be true ${ }^{1}$ for exchange to take place:

$$
O_{0} \cap O_{j}^{c} \neq \varnothing \quad \text { and } \quad O_{0}^{c} \cap O_{j} \neq \emptyset .
$$

If this criterion is met, then peer 0 gets all the segments that peer $j$ has. Similarly, peer $j$ gets all segments that peer 0 has.

A4: All nodes $j \in M$ have only a strict subset of universe $\left(O_{j} \subsetneq N\right)$. Only seed has the universe $N$. Any number of segments can be fetched from the seed without the need for any exchange criterion as in $\mathrm{A} 3$.

\subsection{Problem formulation}

The problem is analysed from the perspective of peer 0 . To begin with, peer 0 has no segments and his objective is to download the universe $N$ at low cost.

${ }^{1} X^{c}$ denotes the complement of set $X$. 
Peer 0 can download segments either from the seed or from peers in $M$. For downloading from the seed, peer 0 needs to pay a cost of $C_{s}$ for each segment, and he need not satisfy the $G$ and $\mathrm{T}$ criterion. For downloading from peer $j \in M$, a cost $C_{j}$ needs to be paid and the $\mathrm{G}$ and $\mathrm{T}$ criterion should be satisfied between the segments sets available with peer 0 and peer $j$ at the time of the exchange.

Let us assume $O_{0, s} \subseteq N$ is fetched from the seed, followed by exchange with peers ${ }^{2}$ in $P_{\text {exc }}$. Hence, the total cost of downloading the universe is given by:

$$
C_{T}=C_{s}\left|O_{0, s}\right|+\sum_{j \in P_{e x c}} C_{j}
$$

The objective of this paper is to determine an optimal $O_{0, s}$ and a set of peers $P_{\text {exc }}$ such that peer 0 has the universe $N$ at the end of all G\&T compliant exchanges with peers in $P_{\text {exc }}$, and the total cost $C_{T}$, is minimized.

\section{Analysis}

Let $O_{0, s} \subseteq N$ be the set of segments that is fetched from the seed. Once $O_{0, s}$ is fetched, peer 0 tries to acquire $N \backslash O_{0, s}$ from other peers $\left(P_{\text {exc }} \subseteq M\right)$ at low cost, while conforming to the G\&T criterion. Total cost for peer 0 is given by $C_{T}=C_{s}\left|O_{0, s}\right|+\sum_{j \in P_{e x c}} C_{j}$. This problem is $\mathcal{N} \mathcal{P}$-hard as proved in Lemma 1.

To progress towards a solution, we make the following observations; proofs are straight forward.

Observation 1: Each peer belonging to a minimum cost set cover has at least one unique segment which is not available with any other peer in the set cover.

Observation 2: From Obs.1, it follows that if peer 0 can exchange with any one peer in a minimum cost set cover, then he can exchange with all others in the set cover as well.

Observation 3: Minimum number of segments to be fetched from the seed to enable peer 0 to fetch all segments is $\max \left(\left|N \backslash \cup_{j \in M} O_{j}\right|, 1\right)$. (If all peers do not cover the universe, then one needs to fetch segments which are not there with any of the peers in M. Otherwise, choosing any $O_{0, s}=\{e\}$ such that $e \in\left(\cap_{j \in P_{s c p}} O_{j}\right)^{c}$ will enable peer 0 to fetch the universe, here $P_{s c p}$ is the standard set cover solution.)

We define instance of our problem as:

Minimum Cost G\&T criterion (MCGT) Problem: Instance defined by $\left(N, M, O_{j}\right.$ $\left.(j \in M), C_{j}(j \in M),, C_{s}\right)$. We need to find $O_{0, s} \subseteq N$ and $P_{e x c} \subseteq M$ such that,

Condition 1: $O_{0, s} \cup\left(\bigcup_{j \in P_{e x c}} O_{j}\right)=N$.

Condition 2: Peer 0 can exchange with peers in $P_{\text {exc }}$ while complying with the G\&T criterion.

\footnotetext{
${ }^{2}$ Peer 0's augmented set will always be considered for exchange. Hence, the order of peers in which exchanges are carried out by peer 0 also matters.
} 
Condition 3: $\left(C_{s}\left|O_{0, s}\right|+\sum_{j \in P_{e x c}} C_{j}\right)$ is minimized.

We define an instance of the Set cover problem as: that,

Instance defined by $\left(\bigcup_{j=1}^{m} O_{j}, M, O_{j}(j \in M), C_{j}(j \in M)\right)$. We need to find $P_{\text {exc }} \subseteq M$ such

Condition 1: $\bigcup_{j \in P_{e x c}} O_{j}=\bigcup_{j \in M} O_{j}$

Condition 2: $\sum_{j \in P_{e x c}} C_{j}$ is minimized.

Lemma 1. The problem of finding the minimum cost solution (i.e., minimizing the cost $C_{T}$ ) for the MCGT problem is NP-hard.

Proof. We use the technique of restriction. Consider instances of the MCGT problem satisfying the following:

(a) There exists an element $e_{0} \in N$, such that $e_{0}$ is missing from each peer's object set, i.e., $e_{0} \notin O_{j}, \forall j \in M$.

(b) $\bigcup_{j \in M} O_{j}=N \backslash\left\{e_{0}\right\}$.

For instance of this type, peer 0 must download $e_{0}$ from the seed, as $e_{0}$ is not available with any peer $j, 1 \leq j \leq m$. With $e_{0}$ in hand, peer 0 is in position to exchange with any peer in $M$ as none of the peers has $e_{0}$.

Then, we set $O_{0, s}=\left\{e_{0}\right\}$, and note that condition 2 is satisfied. Further, we observe that problem of obtaining $P_{\text {exc }}$ such that conditions 1 and 3 are satisfied is the same as a minimum cost set cover problem, with

Set of elements: $N \backslash\left\{e_{0}\right\}=\bigcup_{j \in M} O_{j}$

Subsets: $O_{j}, \forall j \in M$

Costs: $C_{j}, \forall j \in M$.

Hence, by restriction, the MCGT problem is $\mathcal{N} \mathcal{P}$-hard.

Alternatively, in the language of deterministic polynomial-time reduction, we reduce the set cover problem instance $\left(\bigcup_{j=1}^{m} O_{j}, M, O_{j}(j \in M), C_{j}(j \in M)\right)$ to the MCGT problem instance $\left(\bigcup_{j=1}^{m} O_{j} \bigcup e_{0}, M, O_{j}(j \in M), C_{j}(j \in M), C_{s}\right)$, where $e_{0}$ is any element not in $\bigcup_{j=1}^{m} O_{j}$ and $C_{s}=\sum_{j=1}^{m} C_{j}+1$.

This reduction takes only linear time in the input set cover instance $\left(\bigcup_{j=1}^{m} O_{j}, M, O_{j}(j \in\right.$ $\left.M), C_{j}(j \in M)\right)$.

Now observe that $C_{o p t}$ is the minimum cost solution for the set cover problem instance $\left(\bigcup_{j=1}^{m} O_{j}, M, O_{j}(j \in M), C_{j}(j \in M)\right)$ if and only if $C_{T}=C_{s}+C_{o p t}$ is the minimum cost solution for the above-mentioned MCGT problem instance $\left(\bigcup_{j=1}^{m} O_{j} \cup e_{0}, M, O_{j}(j \in M), C_{j}\right.$ $\left.(j \in M), C_{s}\right)$. To see the proof of this, suppose $C_{o p t}$ is the minimum cost solution for the set cover instance and $C_{T}$ is the minimum cost solution for the MCGT instance. Peer 0 must download $e_{0}$ from the seed by paying a cost of $C_{s}$. At this point it has two options - download more objects from the seed or acquire the remaining objects from the other peers at a minimum cost of $C_{o p t}$. But note that if peer 0 downloads any object in addition to $e_{0}$ then it pays an extra $C_{s}=\left(\sum_{j=1}^{m} C_{j}+1\right)$ which is greater than $C_{\text {opt }}$. Hence the optimum strategy for peer 0 is 
to download $e_{0}$ from the seed, followed by acquiring the remaining objects from other peers, making the minimum cost solution for this MCGT instance $C_{s}+C_{o p t}$.

Hence, the set cover problem reduces in deterministic polynomial time to the MCGT problem, implying the latter's NP-hardness.

We move on to obtain the optimal cost for peer 0 .

Based on the segments available with the peers, two cases can occur.

Case 1: All segments not available with the peer group: $\cup_{j=1}^{m} O_{j} \neq N$

In this case, peer 0 has to fetch $N \backslash \bigcup_{j=1}^{m} O_{j}$ from the seed anyway. Since fetching $N \backslash$ $\bigcup_{j=1}^{m} O_{j}$ will enable peer 0 to exchange with all other peers, the optimal value of $O_{0, s}$ is given by $O_{0, s}=N \backslash \bigcup_{j=1}^{m} O_{j}$.

With this $O_{0, s}$, the G\&T criterion is satisfied automatically for all peers in $M$, reducing the problem to a standard set cover problem, $\left[P_{\text {exc }}, C_{\text {peer }}\right]=\operatorname{setcover}\left(\bigcup_{j=1}^{m} O_{j}, M, C\right)$.

Hence, the optimum cost for peer 0 is $C_{T}=\sum_{e \in N \backslash \cup_{j=1}^{m} O_{j}} C_{s}(e)+C_{\text {peer }}$.

Case 2: All segments available with the peer group: $\cup_{j=1}^{m} O_{j}=N$

Suppose that peer 0 fetches segment $e \in N$ from the seed. Let $\Gamma^{*, e}$ represent the optimal cost of acquiring the universe through G\&T-compliant exchanges after having obtained $e$ from the seed $^{3}$. Lemma 2 characterizes the optimal cost for various $e$.

Lemma 2. Let $O_{0, s}=\{e\}$ and $\mathcal{P}_{e, \min }=\left\{P_{e, \min }:\left[P_{e, \min }, C_{e, \min }\right]=\operatorname{setcover}(N \backslash\{e\}, M, C)\right\}$ be all the solution sets for acquiring $N \backslash\{e\}$ from peers in $M$ at the low cost. We define $\tilde{N}_{e}$ as

$$
\tilde{N}_{e}=\left(\bigcap_{P_{e, \min } \in \mathcal{P}_{e, \min }} \bigcap_{j \in P_{e, \min }} O_{j}\right)^{c}
$$

If $e \in \tilde{N}_{e}$, then $\Gamma^{*, e} \leq C_{s}+C_{s c p}$, otherwise, $\Gamma^{*, e}>C_{s}+C_{s c p}$.

Proof. If segment $e$ is fetched from the seed at cost $C_{s}$, then peer 0 will try to acquire $N \backslash\{e\}$ from the other peers. Thus, $\left(C_{s}+C_{e, \min }\right)$ is the low cost of obtaining the universe $N$ when the G\&T criterion is not considered. When the G\&T criterion is taken into account, the low cost $\Gamma^{*, e}$ cannot be any less. Therefore,

$$
\Gamma^{*, e} \geq C_{s}+C_{e, \min } \quad \forall \quad e \in N
$$

In (1), equality will be achieved whenever exchange is possible with at least one peer in $P_{e, \min }$ (by Observation 2) where $P_{e, \min } \in \mathcal{P}_{e, \min }$. This happens when for some $P_{e, \text { min }}$ at least one peer in $P_{e, \min }$ does not possess $e$. Figures $4 \mathrm{a}$ and $4 \mathrm{~b}$ correspond to this scenario.

\footnotetext{
${ }^{3}$ We note that all minimum cost set cover solutions are considered in arriving at $\Gamma^{*, e}$.
} 


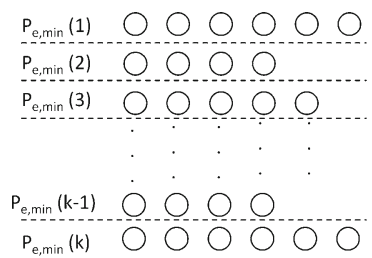

(a) None of the peers have segment $e\left(e \in \tilde{N}_{e}\right)$.

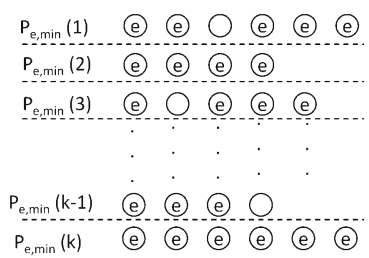

(b) Some of the peers have segment $e\left(e \in \tilde{N}_{e}\right)$.

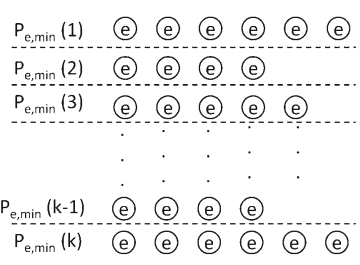

(c) All the peers have segment $e\left(e \notin \tilde{N}_{e}\right)$.

Figure 4. Various possibilities for elements of $\mathcal{P}_{e, \min }$. Each of the circle denotes a peer in $M$. Circles with $e$ shows presence of segment $e$ with that particular peer.

Also, we note that since every cover of $N$ is also a cover of $N \backslash\{e\}$,

$$
\therefore \quad C_{e, \min } \leq C_{s c p} \quad \forall \quad e \in N
$$

If $e \in \tilde{N}_{e}, \exists P_{e, \text { min }} \in \mathcal{P}_{e, \text { min }}$ such that there is at least one peer in $P_{e \text {,min }}$ who does not have segment $e$. Hence, peer 0 can exchange with that particular peer and follow it up by exchanges with other peers in $P_{e, \text { min }}$. Thus, peer 0 can acquire the universe $N$ by means of G\&T compliant exchanges. Figures $4 \mathrm{a}$ and $4 \mathrm{~b}$ help in visualizing this. Therefore, equality will exist in (1):

$$
\Gamma^{*, e}=C_{s}+C_{e, \min } \quad \forall e \in \tilde{N}_{e}
$$

From (2) and (3),

$$
\Gamma^{*, e} \leq C_{s}+C_{s c p} \quad \forall e \in \tilde{N}_{e}
$$

Hence, the first part of the Lemma is proved.

Next, $e \in \tilde{N}_{e}^{c} \Longrightarrow e \in \bigcap_{P_{e, \min } \in \mathcal{P}_{e, \min }} \bigcap_{j \in P_{e, \min }} O_{j}$. This means that all peers in any $P_{e, \min } \in$ $\mathcal{P}_{e, \text { min }}$ have segment $e$ (figure 4c). Exchange is not possible in this case, therefore from (1) we have

$$
\Gamma^{*, e}>C_{s}+C_{e, \min } \quad \forall \quad e \in \tilde{N}_{e}^{c}
$$

If $e \in \tilde{N}_{e}^{c}$, then figure $4 \mathrm{c}$ shows that solution sets $P_{e, \text { min }} \in \mathcal{P}_{e, \text { min }}$ cover $N$ as well. Therefore,

$$
\begin{array}{ll}
C_{e, \min } \geq C_{s c p} & \forall \quad e \in \tilde{N}_{e}^{c} \\
C_{e, \min }=C_{s c p} & \forall \quad e \in \tilde{N}_{e}^{c}
\end{array}
$$

Using (5) and (6), we have

$$
\Gamma^{*, e}>C_{s}+C_{s c p} \quad \forall \quad e \in \tilde{N}_{e}^{c}
$$

Remark 1. For scenarios corresponding to figure $4 a: \bigcup_{j \in P_{e, \min } O_{j}}=N \backslash\{e\} \forall P_{e, \min } \in \mathcal{P}_{e, \min }$. For scenarios corresponding to Figure $4 b, \exists P_{e, \text { min }} \in \mathcal{P}_{e, \text { min }}$ such that $\bigcup_{j \in P_{e, \min }} O_{j}=$ $N$, therefore $C_{e, \min } \geq C_{s c p}$. Using (2), we get $C_{e, \min }=C_{s c p}$. 


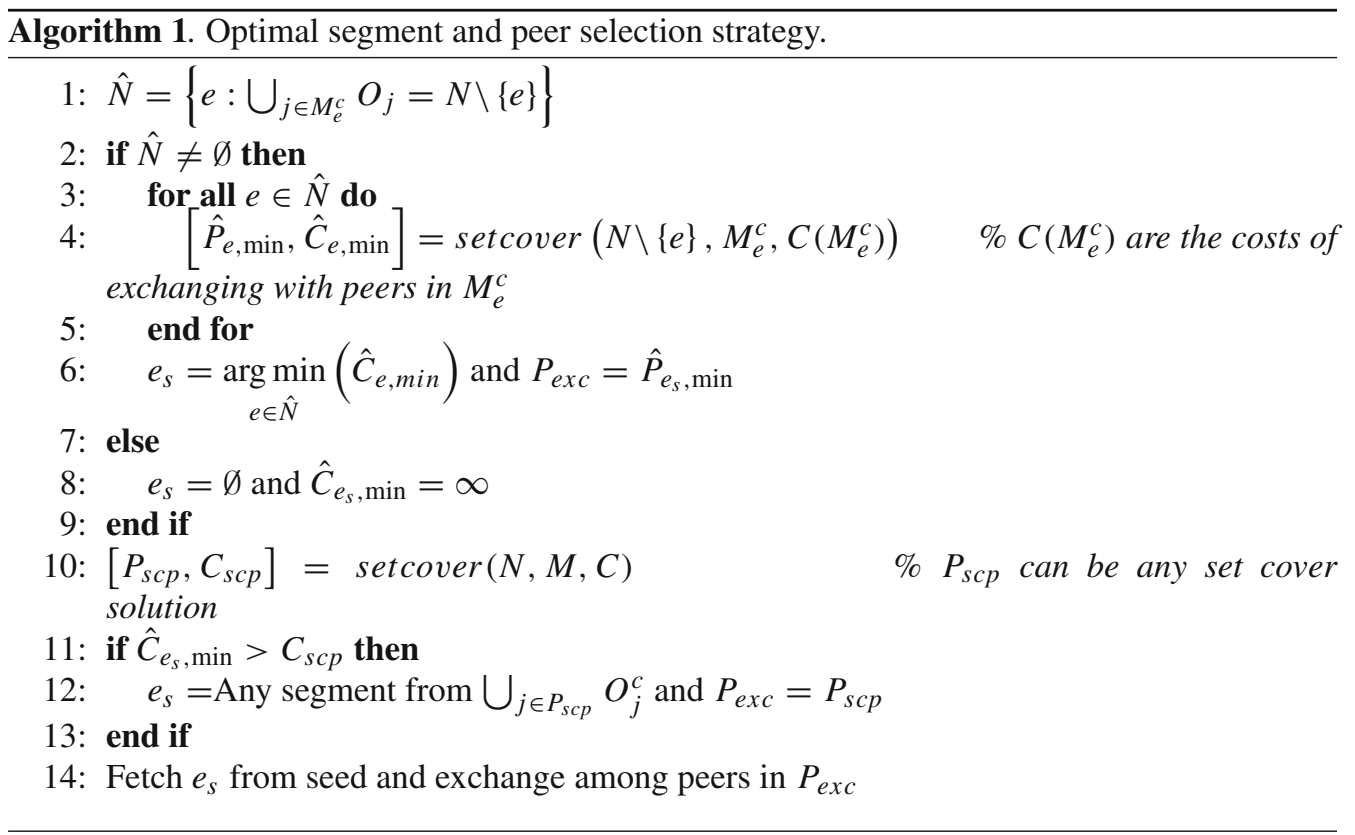

Lemma 2 helps in identifying 'good' candidates for the selection of $O_{0, s}$, and forms the basis of Algorithm 1.

\section{Lemma 3. Algorithm 1 is optimal.}

Proof. By Lemma 2, $O_{0, s}=\{e\}$ can be a optimal choice iff $e \in \tilde{N}_{e}$. Algorithm 1 considers all feasible values (defined by Lemma 2) of $O_{0, s}$ and chooses the segment that minimizes the cost of exchange.

We define $\hat{N}$ as feasible subset of segments fulfilling the condition (for scenarios corresponding to figure 4a) in Line 1 of algorithm.

Let $M_{e} \subseteq M$ be the subset of peers having segment $e$. We note that if $C_{e \text {,min }}$ is strictly less than $C_{s c p}$, then $P_{e, \min } \subseteq M_{e}^{c}, \forall P_{e, \min } \in \mathcal{P}_{e, \min }$.

If $\hat{N} \neq \phi$, we evaluate the cost of acquiring the remaining segments from peers in $M_{e}^{c}$ for each $e \in \hat{N}$ (Lines (3)-(5) of Algorithm 1) and choose $e_{s}$ that minimizes the cost of acquiring the remaining segments. Otherwise, we define $\hat{C}_{e_{s}}$, min as infinity.

If $\hat{C}_{e_{s}, \min }>C_{s c p}$, then $e_{s}$ can be any segment from $\bigcup_{j \in P_{s c p}} O_{j}^{c}$ and exchange with peers $P_{\text {exc }}$. The cost of exchange among peers is $C_{\text {scp }}$.

To summarize, Algorithm 1 first searches for scenarios corresponding to figure $4 \mathrm{a}$ in lines (1)-(9). In case search results in cost of exchange $\hat{C}_{e_{s} \text {, min }}>C_{s c p}$, then optimal cost of exchange among peers is $C_{s c p}$ as being achieved in scenarios corresponding to figure 4b. Hence, Algorithm 1 is optimal.

After fetching $e_{s}$ from seed, peer 0 will initiate exchange with peers in $P_{\text {exc }}$ not having segment $e_{s}$. Now, using the unique elements of these peers (from Obs. 1), peer 0 can exchange with remaining peers in $P_{\text {exc }}$ (from Obs. 2) and get the universe. 
Algorithm 1 is built on a procedure to solve the minimum cost set cover problem. It is known that the latter is $\mathcal{N} \mathcal{P}$-hard. Hence, repeated evaluation of the set cover problems in lines 3 to 5 of Algorithm 1 is computationally most expensive.

Algorithm 2 sacrifices optimality to reduce computation. In Algorithm 2, we solve the problem setcover $(N, M, C)$ only once, and identify the unique elements with each peer in $P_{s c p}$ (line 2 of Algorithm 2). If each peer has at least 2 unique elements, then it suffices to choose $e_{s}$ randomly from $\bigcup_{j \in P_{s c p}} O_{j}^{c}$ (line 4). On the other hand, if one or more peer in $P_{s c p}$ has a single unique element, then we avoid exchanging with the most 'expensive' of such peers (line 7 of Algorithm 2).

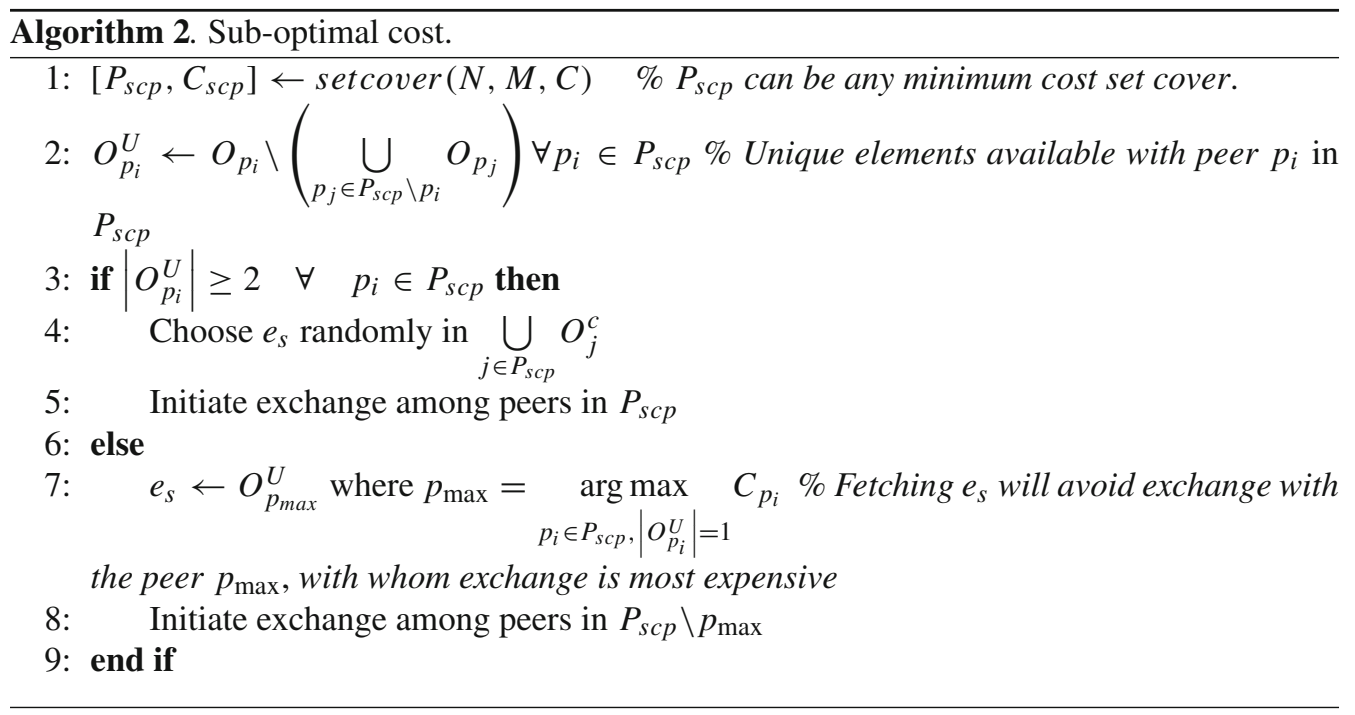

\section{Results}

The objective of this section is to compare the performance of Algorithms 1 and $2^{4}$ with those based on strategies used in the Bittorrent protocol, used widely in peer-to-peer networks. The Bittorrent algorithms are modified slightly so that exchanges satisfy the G\&T criterion. The question we address is: supposing all algorithms allowed only G\&T-compliant exchanges, what can we say about their relative performance?

For selecting the initial set of segments, Bittorrent uses either a random or a Rarest-First approach, followed by the choking of peers, depending on the history of peer activities (Pouwelse et al 2005). The costs $C_{j}$ were assumed to be a function of all parameters of interest to peer 0 . Algorithms 3 and 4 are based on segment and peer selection strategies used in Bittorrent.

Algorithm 3 selects the initial segment and peer uniformly at random. Peering happens if the G\&T criterion is satisfied.

\footnotetext{
${ }^{4}$ For solving set cover problem, we write the same as binary Integer program. We use bintprog function of the Optimization toolbox, MATLAB 2013b for evaluating the solution. Bintprog uses the branch and bound method for solving binary integer program.
} 

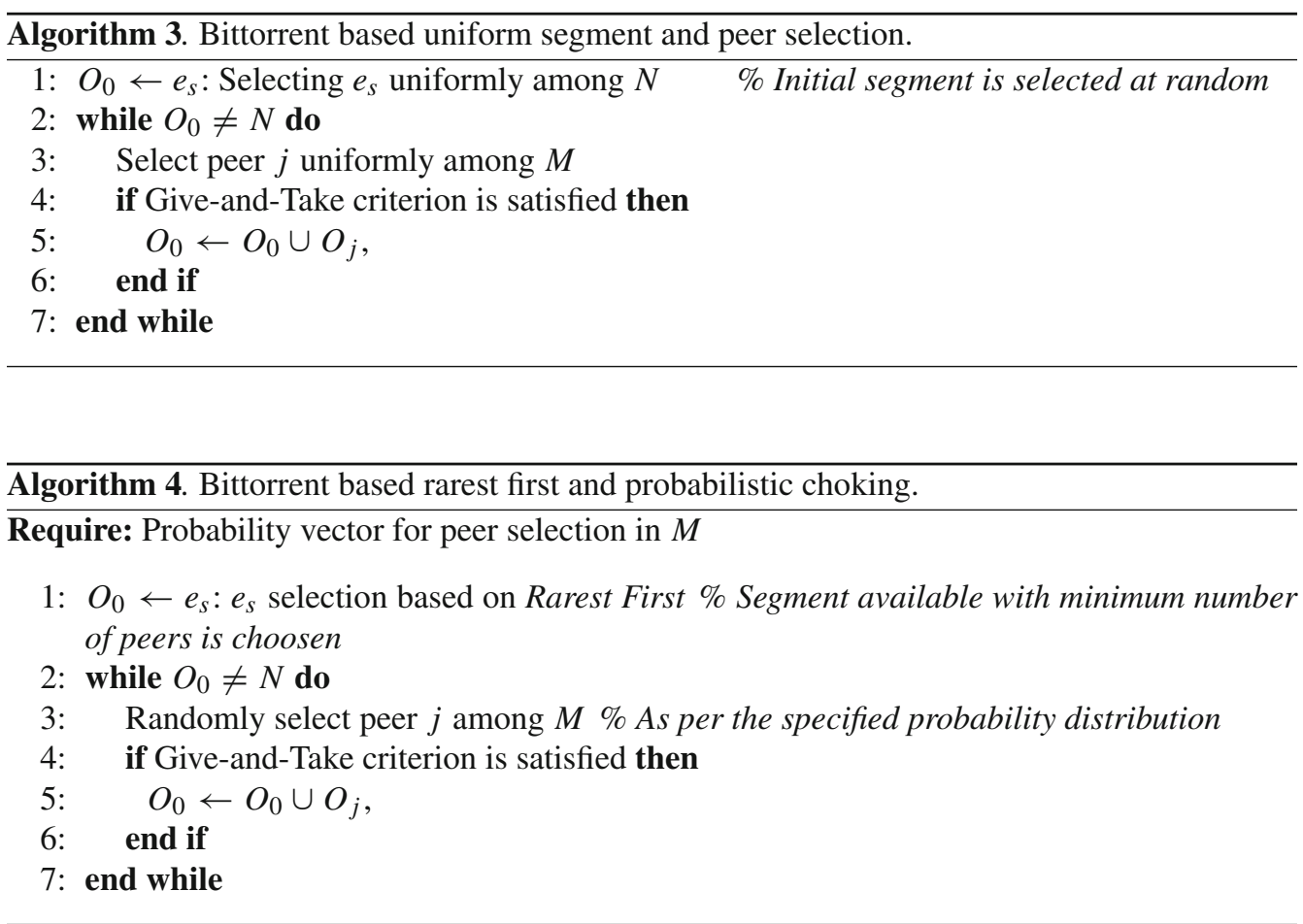

Algorithm 4 uses the Rarest First strategy for selecting the initial segment, followed by peer selection based on probabilities of peer un-choking (Locher et al 2006). First, the segment available with the least number of peers is chosen. Then, one of the peers is unchoked, based on their unchoking probabilities.

For simulation purposes, the probability vectors in Algorithm 4 were defined/generated as follows:

4a: Uniformly at random

4b: At random, but with low probability values assigned to peers with high cost

4c: Prob (peer $j$ is selected $)=\frac{1-\frac{C_{j}}{\sum_{j \in M} C_{j}}}{m-1}$. This form ensures that peers with high cost are chosen
with low probability.

The results are shown in figure 5. $|M|=m$ denotes the number of peers and $|N|=n$ the size of the universe. For $j \in M$, the segment set $O_{j}$ and the cost of exchanging segments with the peer were generated uniformly at random. For each set of bars, the results shown are averages over 1000 sample runs. Algorithm 2 sacrifices optimality for shorter running times, but achieves costs that are very close to optimal. It can be seen that Algorithm 1, which is optimal, achieves much lower cost-on an average 51\%,51\%, 42\% and 51\% over all sets in figure 5, respectively, than algorithms 3, 4a, $4 \mathrm{~b}$ and $4 \mathrm{c}$.

Figure 6 plots the cost of exchange against the time taken for running each algorithm on 50 randomly generated data points, with 300 segments and 200 peers. From the figure, one can 


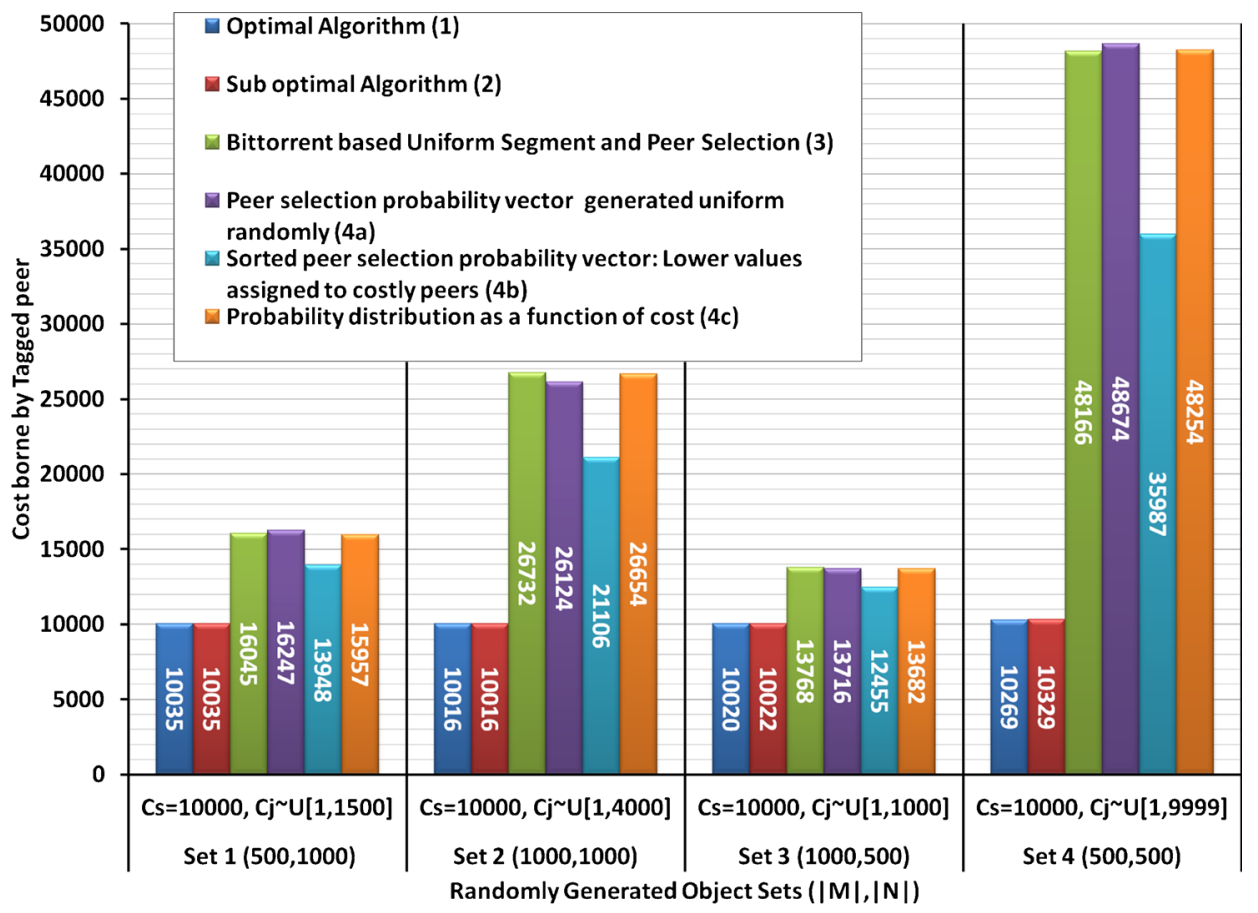

Figure 5. For all sets, $\bigcup_{j \in M} O_{j}=N$; Each set is generated independently shows the cost of acquiring the universe for a sample path. For algorithms $3,4 a, 4 b$ and $4 \mathrm{c}$ ), the same has been averaged over 1000 runs.

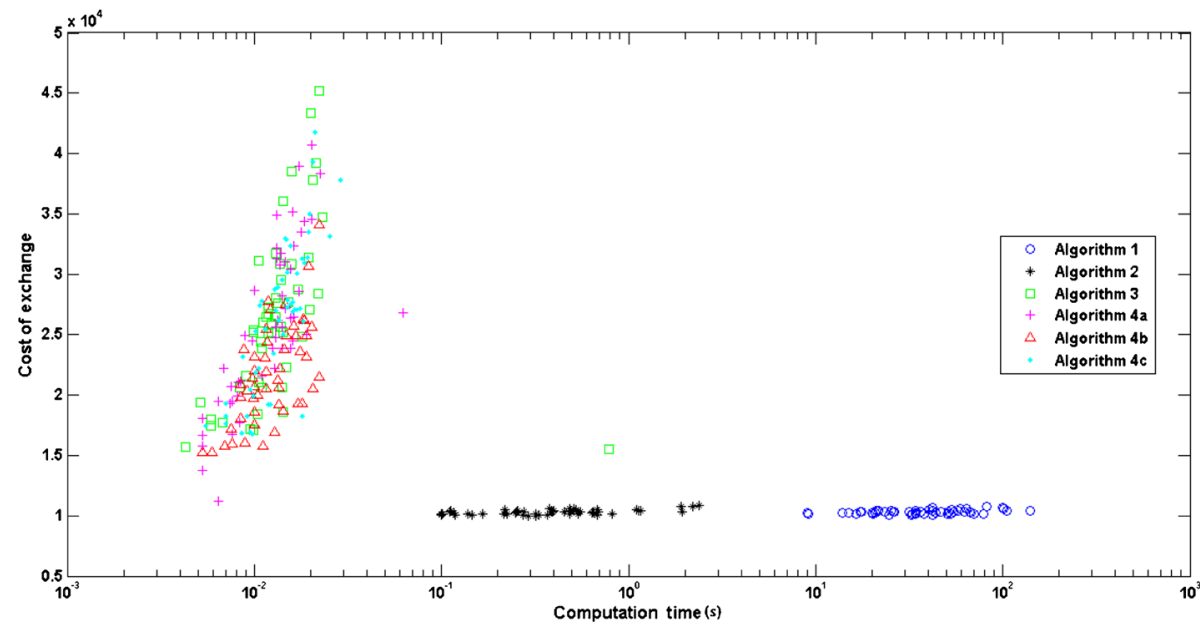

Figure 6. Trade-off between computation time and cost for 50 randomly generated datasets with 300 segments and 200 peers. Cost against the time taken for running each algorithm on each of data point is plotted. 
Table 1. Mean running time for algorithm 2 (on MAT$\mathrm{LAB}$ ) for different sets of values of $m$ and $n$ averaged over 500 randomly generated object sets (Machine Specifica-

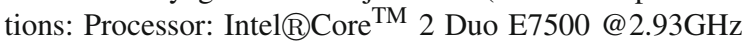
(2CPUs); Memory: 2004 MB RAM).

\begin{tabular}{lcc}
\hline $\begin{array}{l}\text { Number of } \\
\text { peers }(\mathrm{m})\end{array}$ & $\begin{array}{c}\text { Number of } \\
\text { Segments(n) }\end{array}$ & $\begin{array}{c}\text { Mean running time } \\
\text { for algorithm 2 }\end{array}$ \\
\hline 30 & 50 & $0.0298 \mathrm{~s}$ \\
100 & 100 & $0.0967 \mathrm{~s}$ \\
150 & 200 & $0.2809 \mathrm{~s}$ \\
200 & 300 & $0.727 \mathrm{~s}$ \\
300 & 400 & $1.1324 \mathrm{~s}$ \\
500 & 400 & $2.0078 \mathrm{~s}$ \\
500 & 600 & $4.8460 \mathrm{~s}$ \\
600 & 600 & $5.7293 \mathrm{~s}$ \\
700 & 700 & $16.9082 \mathrm{~s}$ \\
800 & 800 & $20.2471 \mathrm{~s}$ \\
\hline
\end{tabular}

observe that costs achieved by both Algorithms 1 and 2 are quite similar, but the running time of Algorithm 2 is orders of magnitude ${ }^{5}$ lower than that of Algorithm 1.

Also, each of the Algorithms 3, 4a, 4b and 4c runs very quickly, but the cost of exchange is higher than that achieved by Algorithms 1 and 2. To summarize, the proposed Algorithm 2 guarantees the best cost of exchange possible, at the expense of roughly one and a half orders of magnitude increase in computation time.

Is Algorithm 2 suitable for practical applications?

Algorithm 2 is built over a procedure to solve an $\mathcal{N} \mathcal{P}-$ hard problem. Hence, running time of Algorithm 2 increases drastically as the number of peers or segments is increased. Still, Algorithm 2 can be a practical choice in many situations.

For instance, table 1 shows the mean running time of Algorithm 2 for 500 randomly generated object sets. We compare the observed running times of Algorithm 2 with the reported observations in Sharma et al (2013). Visual observations from figure 1 in Sharma et al (2013) show that for 10 peers, the system takes around 10-30 s for getting hold of the first chunk. Hence, Algorithm 2 can be a good alternative for coming up with a list of peers, as its running time is below $30 \mathrm{sec}$ for systems having many more than 10 peers. Moreover, it achieves near-optimal cost.

Also, Liao et al (2007) reports download completion time of 10000-40000 seconds for different scenarios. Hence, we believe it is quite acceptable for the peer to spend 20 seconds - which is negligible compared to the download completion time-in an initial computation that yields nearly the best possible download cost.

\section{Conclusions and future work}

In this paper, our objective was to devise a mechanism that would discourage free riding in P2P CDNs. To this end, we introduced the G\&T criterion. Subsequently, we have analysed a

\footnotetext{
${ }^{5}$ The complexity for Algorithm 1 and Algorithm 2 is $O\left(n 2^{m}\right)$ and $O\left(2^{m}\right)$ respectively.
} 
problem of interest to a newly-arrived peer-that of acquiring the universe, scattered among the segment sets of peers-at minimal cost, and in conformance with the G\&T criterion. Our analyses suggested the optimal algorithm. Performance comparison with some commonly used algorithms quantified the extent of improvement possible. We also studied the computation time for prescribing the set of segment and peer selection strategies to the newly arrived peer. For moderate-sized problems, algorithm 2 can lead to near-optimal cost while the running time remains within a few seconds; thus, algorithm 2 emerges as a practical option when the problem size is not too big. For larger problem sizes, our approach indicates the extent of improvement in cost that is possible. This indicates the need to devise algorithms that reduce the large gap between optimal cost and the cost achieved by Bittorrent-like algorithms; we would like to take up this as future work.

Further, in the present paper, we considered one new peer acquiring segments, while other peers' segment sets were 'frozen'. We are in the process of studying dynamic scenarios in which the system evolves with time. In future, we also would like to study the situation that arises when all peers are involved in exchanges simultaneously.

\section{References}

Aggarwal V, Feldmann A and Scheideler C 2007 Can isps and p2p users cooperate for improved performance? SIGCOMM Comput. Commun. Rev. 37: 29-40

Alon N, Moshkovitz D and Safra S 2006 Algorithmic construction of sets for k-restrictions. ACM Trans. Algorithms 2: 153-177

Caprara A, Toth P and Fischetti M 2000 Algorithms for the set covering problem. Ann. Oper. Res. 98: 353-371

Choffnes D R and Bustamante F E 2008 Taming the torrent: a practical approach to reducing cross-isp traffic in peer-to-peer systems. IGCOMM Comput. Commun. Rev. 38: 363-374

Chvatal V 1979 A greedy heuristic for the set-covering problem. Math. Oper. Res. 4: 233-235

Feige U 1998 A threshold of $\ln n$ for approximating set cover. J. ACM 45: 634-652

Feldman M and Chuang J 2005 Overcoming free-riding behavior in peer-to-peer systems. SIGecom Exch. 5: 41-50

Feldman M, Papadimitriou C, Chuang J and Stoica I 2006 Free-riding and whitewashing in peer-to-peer systems, pp. 1010-1019

Karakaya M, Korpeoglu I and Ulusoy O 2009 Free riding in peer-to-peer networks. Internet Comput. IEEE, 13: 92-98

Liao W C, Papadopoulos F and Psounis K 2007 Performance analysis of bittorrent-like systems with heterogeneous users. Perform. Eval. 64: 876-891

Locher T, Moor P, Schmid S and Wattenhofer R 2006 Free Riding in BitTorrent is Cheap, in: Fifth Workshop on Hot Topics in Networks (HotNets-V), Irvine, CA, US

Menasché D S, Rocha A A A, de Souza e Silva E A, Leão R M, Towsley D and Venkataramani A 2010 Estimating self-sustainability in peer-to-peer swarming systems. Perform. Eval. 67: 1243-1258

Nishida H and Nguyen T 2010 A global contribution approach to maintain fairness in p2p networks. IEEE Trans. Parallel Distrib. Syst., 21: 812-826

Plissonneau L, Costeux J L and Brown P 2005 Analysis of peer-to-peer traffic on adsl, in: Dovrolis, C (Ed.), Passive and Active Network Measurement. Berlin, Heidelberg: Springer. volume 3431 of Lecture Notes in Computer Science, pp. 69-82

Pouwelse J, Garbacki P, Epema D and Sips H 2005 The bittorrent p2p file-sharing system: Measurements and analysis, in: Castro, M., Renesse, R. (Eds.), Peer-to-Peer Systems IV. Springer Berlin Heidelberg. volume 3640 of Lecture Notes in Computer Science, pp. 205-216 
Rahman R, Meulpolder M, Hales D, Pouwelse J, Epema D and Sips H 2010 Improving efficiency and fairness in $\mathrm{p} 2 \mathrm{p}$ systems with effort-based incentives, In: Communications (ICC), 2010 IEEE International Conference on, pp. 1-5

Sharma P, Bhakuni A and Kaushal R 2013 Performance analysis of bittorrent protocol, In: National Conference on Communications (NCC), 2013, pp. 1-5

Typpi T 2009 Game theory in peer-to-peer networks, in: Seminar on Internetworking, Spring 2009

Vakali A and Pallis G 2003 Content delivery networks: status and trends. Internet Comput. IEEE 7: 68-74 